\title{
Transcriptomic profiling of long non-coding RNAs in hepatitis B virus-related hepatocellular carcinoma
}

\author{
Hongxia Fan ${ }^{1}$, Qiaoge Zhang ${ }^{1}$, Xiaopei Zhao ${ }^{1}$, Ping Lv ${ }^{1}$, Min Liu ${ }^{1}$ and Hua Tang ${ }^{1}$ \\ ${ }^{1}$ Tianjin Life Science Research Center and Department of Pathogen Biology, School of Basic Medical Sciences, Tianjin Medical \\ University, Tianjin, China
}

Correspondence to: Hua Tang, email: htang2002@yahoo.com, tangh@tmu.edu.cn

Keywords: HBV, long non-coding RNAs, hepatocellular carcinoma, RNA sequencing, $n 346077$

Received: February 03, 2017 Accepted: June 12, $2017 \quad$ Published: June 30, 2017

Copyright: Fan et al. This is an open-access article distributed under the terms of the Creative Commons Attribution License 3.0 (CC BY 3.0), which permits unrestricted use, distribution, and reproduction in any medium, provided the original author and source are credited.

\section{ABSTRACT}

Long non-coding RNAs (IncRNAs) have been reported to be involved in the development and progression of hepatocellular carcinoma (HCC). However, few studies have focus on the dyregulation and the role of IncRNAs in HBV-related HCC. We performed a comprehensive analysis of IncRNAs expression profile in HBV-related HCC tissues samples using deep sequencing. We revealed that a total of 1242 IncRNA transcripts (983 up-regulated and 259 down-regulated) and 1841 mRNA transcripts were significantly differentially expressed in HBV-related HCC patients. Pathway and gene ontology analysis showed that they are involved in the biological process related to HCC development by cis-regulation of co-expressed protein-coding genes. 10 candidate IncRNAs were selected and validated with quantitative real-time PCR analysis. Furthermore, we found that one of most down-regulated IncRNAs, n346077, could suppress HCC cells invasion and migration in vitro. Our findings provide an overview of aberrantly expressed InCRNAs in HBV-related HCC and will be useful for further functional studies of IncRNAs in HBV-related pathogenesis.

\section{INTRODUCTION}

$\mathrm{HCC}$ is one of the most common cancers and is the second leading cause of cancer-related mortality worldwide [1]. The risk factors accounting for HCC development include chronic HBV infection [2], hepatitis $\mathrm{C}$ virus infection [3], excessive consumption of alcohol [4], and aflatoxin exposure [5]. Among them, $\mathrm{HBV}$ is a major etiological agent of HCCs. HBV contributes to HCC formation and development through direct and indirect pathways. The prolonged expression of the viral regulatory protein $\mathrm{HBx}$ and/or altered versions of the preS/S envelope proteins deregulate cell transcription, alter cells proliferation control and sensitize liver cells to carcinogenic factors. The integration of HBV DNA into the host genome induces genomic instability and eventually direct insertional mutagenesis of diverse cancer-related genes $[6,7]$. But the detailed mechanism underlying $\mathrm{HBV}$ induced-HCC is still unclear.

The recent developments in deep sequencing technologies have led to the detection of non-coding RNAs which comprised the majority of the transcriptome. Noncoding RNAs can be generally divided into two classes according to their size: small non-coding RNAs (e.g., microRNAs) and long non-coding RNAs (e.g., lncRNAs) $[8,9]$. lncRNAs are a group of non-coding RNAs longer than 200 nucleotides in length with little or no proteincoding capacity. According to the proximity to nearby coding genes, lncRNAs could be categorized to 5 classes: sense, antisense, intergenic, bidirectional and intronic [9]. IncRNAs can promote/suppress gene expression at epigenetic, transcriptional, and post-translational levels [10], and are involved in various biological processes across every branch of life including cell growth, differentiation, proliferation, survival, migration and so on [10]. Notably, emerging data have showed that the mutation and dysregulation of lncRNAs can result in aberrant expression of gene products that contribute to the progress of various human diseases, especially cancer $[10,11]$. Several lncRNAs have been found to contribute to the pathologic process of HCC by multiple mechanisms $[12,13]$. Among them, the lncRNAs highly up-regulated 
in liver cancer (HULC), high expression in HCC (HEIH), HBx-interspersed nuclear element 1 (HBx-LINE1), H19, HOTAIR, Unigene56159, down-regulated expression by HBx (DREH) and microvascular invasion in HCC (MVIH) are strongly implicated in the development of HBV-related HCC [14-21]. For example, the lncRNA HEIH was highly expressed in the patients with HBVrelated HCC. It promoted cell proliferation through repressing the targets genes of enhancer of zeste homolog 2 (EZH2) [15]. HBx-LINE1 was a viral-human chimeric transcript identified from transcriptome sequencing of $\mathrm{HBV}$-positive HCC cell lines. It functions like a long noncoding RNA to promote HCC through activating Wnt/ $\beta$ catenin signaling pathway [16]. In our recent study, we found that Unigene56159, which is highly expressed in HBV-related HCC tissues, promoted HCC cells migration and invasion by acting as a competing endogenous RNA (ceRNA) for miR-140-5p to de-repress the expression of the target gene Slug [19]. However, few studies have focus on the lncRNA expression profile in HBV-related HCC and the exact role of IncRNAs in HBV-related HCC has not yet been clearly clarified.

In the present study, to identify the IncRNAs that are participated in the progress of $\mathrm{HBV}$-related $\mathrm{HCC}$, we investigated the lncRNA expression profile in $\mathrm{HBV}$-positive $\mathrm{HCC}(\mathrm{HBV}(+) \mathrm{HCC}$ ) and HBV-negative (HBV (-) HCC) using high-throughput RNA sequencing (RNA-Seq) and annotated their functions with gene ontology (GO) analysis and Kyoto Encyclopedia of Genes and Genomes (KEGG) pathway analysis of their cis- and trans-regulated proteincoding genes. Furthermore, the function of n346077, a lncRNA was markedly down-regulated in $\mathrm{HBV}(+) \mathrm{HCC}$, was characterized in vitro. To the best of our knowledge, this is the first report of a comprehensive identification of lncRNAs in HBV-related HCC using RNA-seq analysis, which will facilitate our understanding of the roles of lncRNAs in the development of HBV-related HCC.

\section{RESULTS}

\section{RNA-seq analysis and identification of mRNAs and IncRNAs in HBV-related HCC}

To comprehensively identify lncRNAs and mRNAs associated with HBV-related HCC, we performed the whole transcriptome strand-specific RNA-Seq on rRNAdepleted RNAs from $3 \mathrm{HBV}(+) \mathrm{HCC}$ and $3 \mathrm{HBV}(-)$ HCC tissue samples. A total of 199.4 million clean reads were obtained from sequencing. More than 179.7 million read pairs $(90.1 \%)$ were aligned to the human genome (hg19). The mapped reads were used for assembling putative lncRNAs with cufflink program and the assembled transcripts were annotated. For mRNAs, RefSeq database (Build 38) was chosen as the annotation reference. For lncRNAs, NONCODE v3 database was chosen as the annotation reference. Through the transcripts identification pipeline (Figure 1), we obtained $21841 \mathrm{mRNA}$ isoforms derived from 16662 gene loci and 23326 expressed lncRNA isoforms derived from 23289 gene loci. Furthermore, 8794 $(37.60 \%)$ of all $23326 \mathrm{lncRNAs}$ were not identified in the NONCODE v3 lncRNA annotation.

We characterized the basic features of the lncRNAs and compared them with protein-coding genes where appropriate. Based on their relative genomic locations to

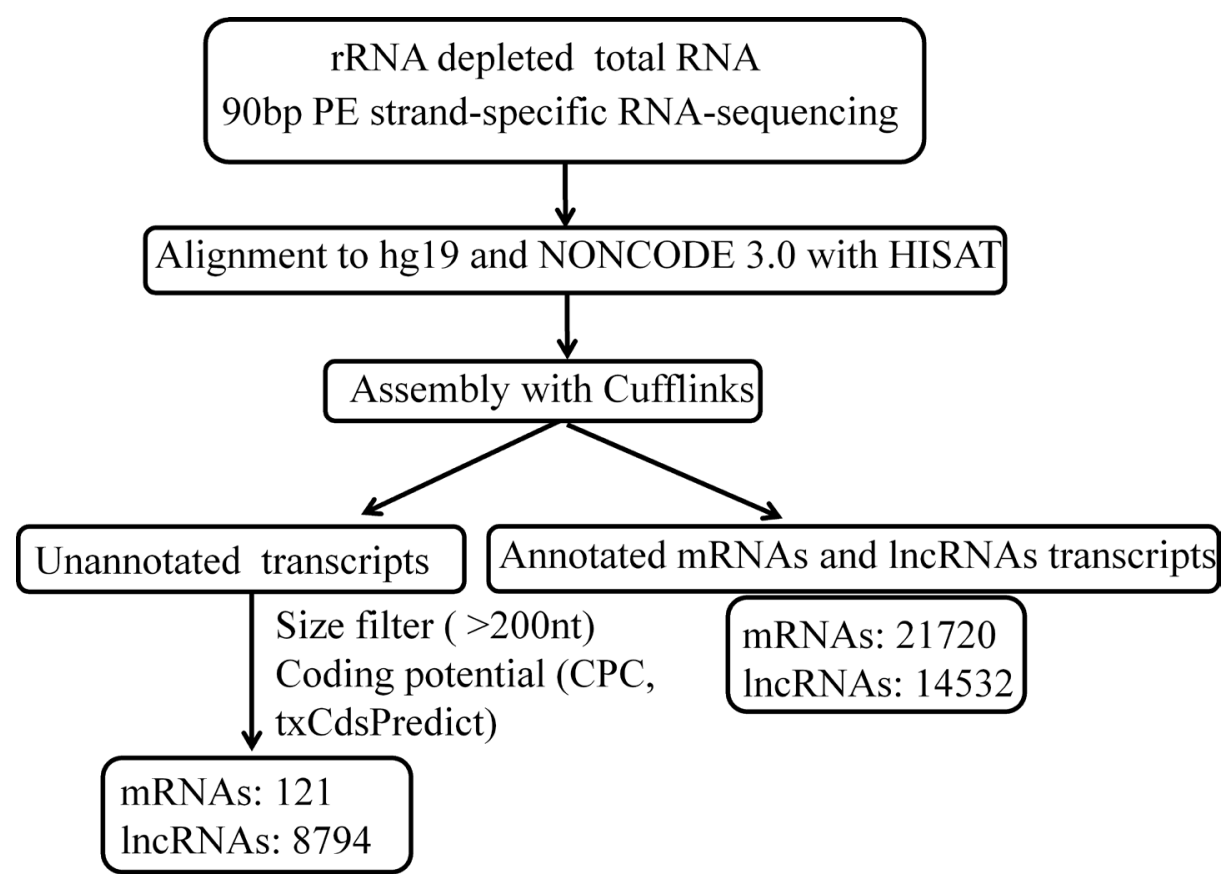

Figure 1: The pipeline for systematic identification of IncRNAs and mRNAs in HBV-related HCC. CPC, Coding Potential Calculator. 
neighboring coding genes, the lncRNAs include 15506 long intergenic lncRNAs, 1277 intronic lncRNAs, 2179 antisense lncRNAs and 4364 others (Figure 2A). Our data showed that the average lncRNAs expression levels were slightly higher than protein-coding gene expression levels (Figure 2B). As shown in Figure 2C, lncRNA transcripts are shorter in length than protein coding transcripts. Moreover, the exons number of IncRNAs was also less than that of mRNAs (Figure 2D). Both lncRNAs and mRNAs were alternatively spliced. On average, there were 1.01 isoforms per lncRNA gene loci and 3.49 isoforms per mRNA gene loci (Figure 2E).

\section{Differentially expressed mRNAs and IncRNAs in HBV-related HCC}

Volcano plot, scatter plots and hierarchical clustering analysis were performed to assess gene expression variation between $\mathrm{HBV}(+) \mathrm{HCC}$ tissues and HBV (-) HCC tissues (Figure 3). IncRNAs and mRNAs with at least 2 fold expression change were identified as significantly differently expressed $(p<0.05, \mathrm{FDR} \leq 0.001)$. Data analysis revealed 1242 lncRNA transcripts and 1841 mRNA transcripts were differentially expressed in HBV (+) HCC tissues compared to HBV (-) HCC tissues. Of the 1242 differentially expressed lncRNA transcripts, 983 were up-regulated and 259 were down-regulated. Of the 1841 differentially expressed mRNA transcripts, 1191 were up-regulated and 650 were down-regulated.

\section{Validation of dysregulated IncRNA expression in HBV (+) HCC patients and HBV (+) cell line}

To validate our RNA-seq data, we randomly selected 5 up-regulated and 5 down-regulated IncRNAs and analyzed their expression levels in another independent cohort of $10 \mathrm{HBV}(+) \mathrm{HCC}$ patients and in HepG2.2.15 cells containing dimers of HBV genomic sequence that could constitutively produce HBV particles with quantitative real-time PCR (RT-qPCR). As shown in Figure 4, compared to HBV (-) HCC, the selected lncRNAs displayed the same expression trend with the RNA-Seq data. Similar result was observed in HepG2.2.15 cells. These data verified the reliability of the RNA-Seq results.

\section{Functional annotation of differentially expressed mRNAs}

GO analysis for the differentially expressed mRNAs was performed to identify their function. The top 10 enriched GO items were listed in Figure 5A, including cell, cell part, binding, cellular process, organelle, metabolic process, biological regulation, organelle part, catalytic activity, regulation of biological process. The KEGG pathway analysis revealed that the most enriched pathways included RNA degradation, fatty acid degradation, chronic myeloid leukemia and metabolic pathway (Figure 5B).

\section{Prediction of IncRNA function}

To evaluate the function of differentially expressed lncRNAs, we predicted their cis-regulated target genes by search for protein-coding genes 10 and $100 \mathrm{~kb}$ upstream and downstream of the lncRNAs, respectively. And then performed GO and KEGG analysis on these cis-regulated target genes. GO analysis demonstrated that the significantly over-represented terms are similar to that for differentially expressed mRNAs (Figure 6A). KEGG pathway analysis showed that these cis-regulated target genes were enriched in metabolism of xenobiotics by cytochrome P450, drug metabolism - cytochrome P450, chemical carcinogenesis, steroid hormone biosynthesis, retinol metabolism, metabolic pathways, fatty acid degradation, glycolysis/gluconeogenesis, complement and coagulation cascades and primary bile acid biosynthesis (Figure 6B). Surprisingly, based on our prediction criterion, there was no trans-regulated target gene for the differentially expressed lncRNAs.

\section{IncRNA n346077 suppresses HCC cells migration and invasion}

n346077, which encodes a 2609bp transcript and located in the opposite strand of mitochondrial ribosomal protein L23 (MRPL23) gene on chromosome 11, is one of the down-regulated lncRNAs, to probe the potential role of it in HCC cells, we first performed MTT, colony formation, cell migration, and invasiveness assays in HepG2 and QGY7703 cells with $\mathrm{n} 346077$ overexpression. The efficiency of the n346077 overexpression plasmid was confirmed in HepG2 and QGY-7703 cells (Figure 7A). As shown in Figure 7B-7D, there was no significant difference in the viability and colon formation ability of HepG2 and QGY7703 cells when n346077 was overexpressed compared to control. But the results of transwell assay with or without Matrigel showed that the cell migration and invasion abilities of both HepG2 and QGY-7703 cells were significantly suppressed after transfection with n346077 (Figure 7E and Figure 7F). These data indicated that $\mathrm{n} 346077$ suppresses cell invasion and migration of HCC cells.

We then examined the effect of n346077 knockdown on the malignant behavior of these two HCC cells. The knockdown efficiency of the pshR-n346077 was confirmed in HepG2 and QGY-7703 cells (Figure 8A). As shown in Figure 8B-8D, knockdown of n346077 has no effect on the viability and colon formation ability of HepG2 and QGY7703 cells. But the cell migration and invasion abilities of both HepG2 and QGY-7703 cells were significantly increased when the expression level of n346077 was decreased (Figure 8E and Figure 8F). These results further confirmed its tumor suppressor role in HCC cells. 


\section{DISCUSSION}

With the emerging studies focusing on the regulatory role of lncRNAs, it has been found that lncRNA contributes significantly to the pathogenesis and progression of cancers, including HCC $[11,12]$. For HBVrelated $\mathrm{HCC}$, previous studies are mainly focus on proteincoding genes and miRNAs, the studies on the deregulated expression of lncRNAs and the involvement of lncRNAs just begin. Pan et al. and Yu et al. analyzed the lncRNA expression profiles between HBV-related HCC tissues
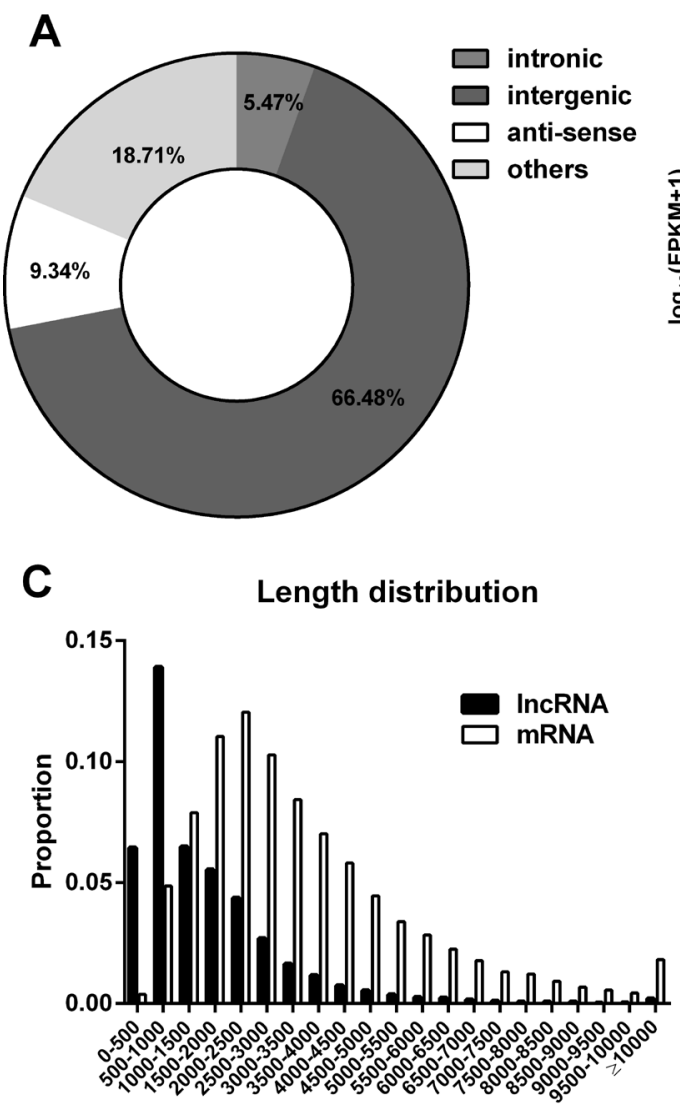

Length (nt)

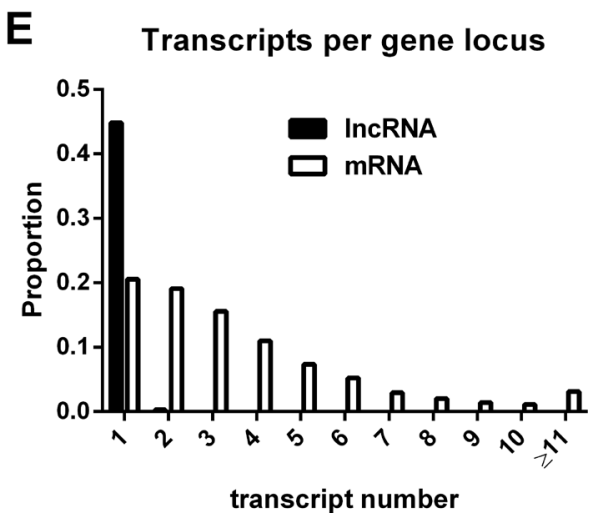

and corresponding normal liver tissues using microarrays without characterizing the function of a specific IncRNA, respectively [22, 23]. Gong et al. investigated lncRNA expression profiles in HBV-related HCC by annotating and analyzing microarray datasets and identified $182 \mathrm{lncRNAs}$ that were specifically differentially expressed in HBVrelated HCC [24]. A recent study reported the expression profile on lncRNA among 5 male HBV-induced HCC cases by using microarray analysis [25]. Yang et al. identified the differentially expressed lncRNAs between HBVrelated HCC and paired peritumoral tissues by microarray
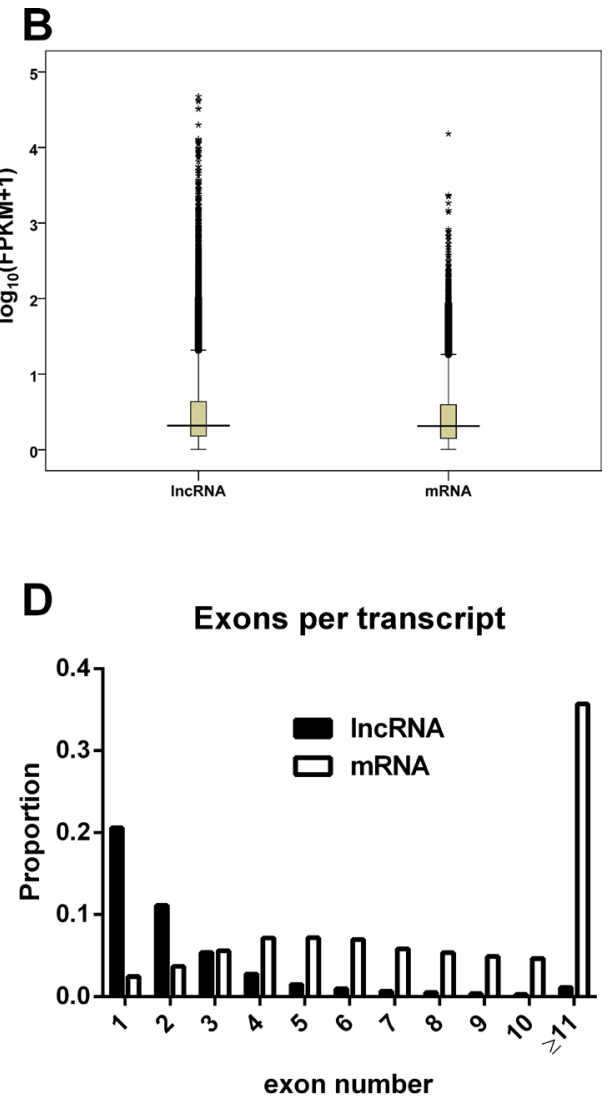
and characterized the oncogenic role of lncRNA-HEIH in tumor progression [15]. However, to our knowledge, systematic characterization of lncRNA in HBV-related HCC is rare. In this study, we generated global lncRNA expression profiles associated with HBV-related HCC using next-generation RNA-seq, which may provide valuable resources to further study the role of lncRNAs in HBV-related HCC development.

As for the first study of genome-wide analysis of lncRNAs expression profiles in HBV-related HCC, we obtained 8757 novel lncRNAs and 14532 known lncRNAs included in NONCODE v3.0 database. The high ratio of
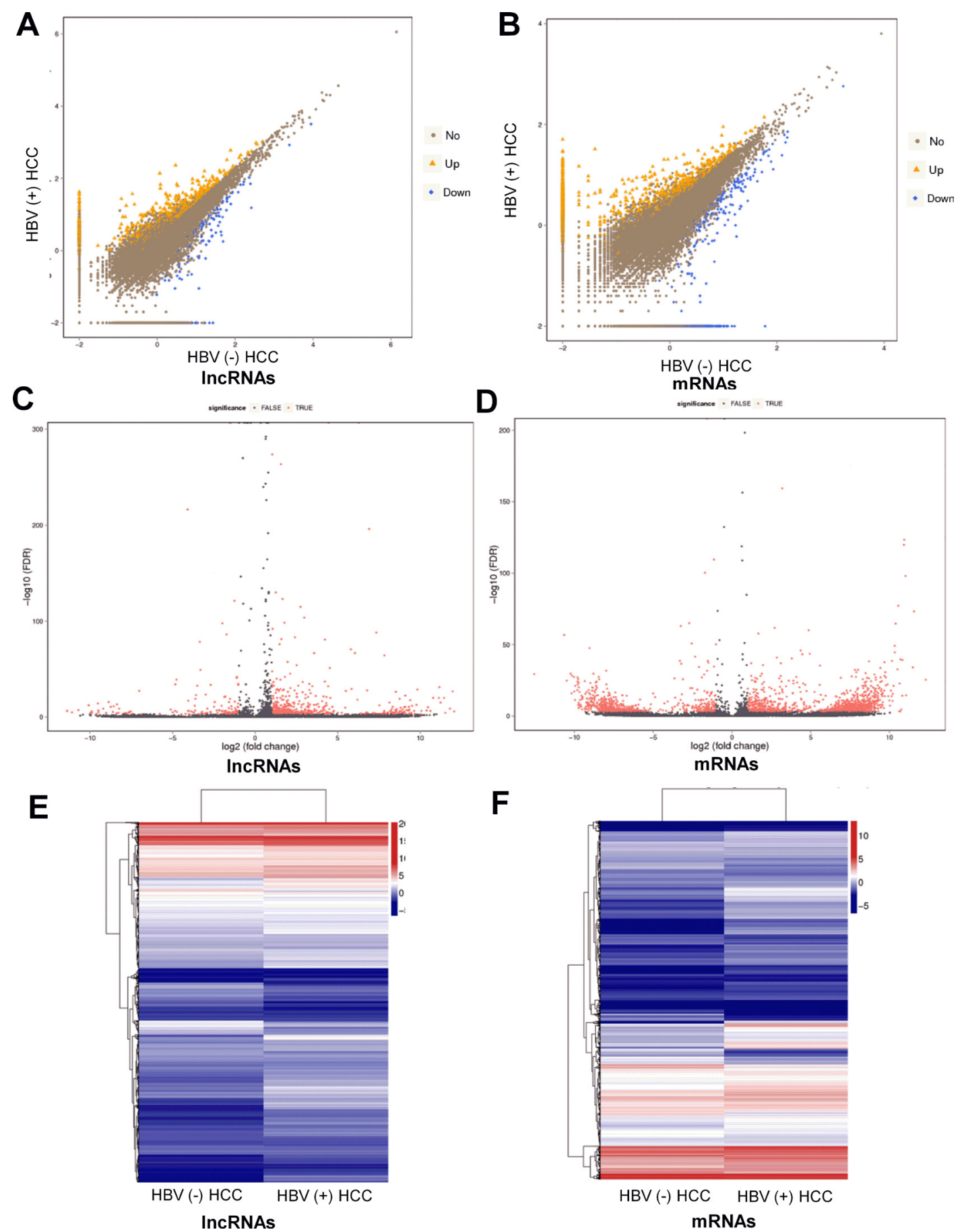

Figure 3: Differentially expressed IncRNAs and mRNAs between HBV (+) HCC and HBV (-) HCC tissue samples. (A) and (B) Scatter plots of lncRNA and mRNA transcripts expression. The values of X and Y axes are the normalized signal values of groups ( $\log 2$ scaled). The symbol triangle and diamond indicated more than 2 fold change between two groups. (C) and (D) Volcano plot of differentially expressed lncRNAs and mRNAs transcripts. The red points represent the differentially expressed genes with statistical significance. (E) and (F) Hierarchical clustering of the expression profiles of differentially expressed lncRNAs and mRNAs transcripts. Red indicates high relative expression and blue low relative expression. 
novel to known lncRNAs may be due to the rarity of studies of the lncRNAs in HBV-related liver diseases. Further, we identified approximately 654 lncRNAs and 515 mRNAs were differentially expressed in HBV-related HCC. We compared the differentially expressed mRNA and lncRNA with previous studies. For example, BAIAP2-AS1 [24], THBS4 [25] are also over-expressed in our samples, which supports the reliability of our study. Although the difference of BAIAP2-AS1 expression did not reach statistical significance, which may be due to the small number of samples analyzed. Moreover, the RT-qPCR results in an independent $\mathrm{HBV}(+) \mathrm{HCC}$ patients and HepG2.2.15 cells showed the same trends of changes in expression levels with the RNA-Seq results, further confirming the accuracy and reliability of our study. We will confirm our RNA-Seq data in larger cohorts of wellcontrolled subjects in subsequent research.

In our study, we find that the average expression level of lncRNA transcripts is slightly higher than that of proteincoding genes (Figure 2B). Similar with our findings, a recent study which focus on the effect of HSV-1 on lncRNAs expression showed that the expression levels of lncRNAs are higher than protein-coding genes after HSV-1 infection for 6 and $8 \mathrm{~h}$, which may be due to the degradation of host mRNAs by viral protein UL14 [26-28]. But lncRNAs are generally lower expressed than protein-coding genes by comprehensive analysis of their expression in multiple human organs [8]. The reason for the difference may be due to the expression of some of the HBV proteins, such as $\mathrm{HBx}$, which has been reported to regulate the expression of many protein coding genes and non-coding RNAs [29]. It may also be due to the HBV DNA integration, which leads to $23.1 \%$ of protein-coding genes and $24.7 \%$ of $\operatorname{lnc}$ RNAs genes with more than two times change $[16,30]$. We will explore the specific mechanism in the future.

Furthermore, we explored the potential function of dysregulated lncRNAs by prediction of their cis- and trans-regulated target genes and subsequently subject them into GO and KEGG analysis. Interestingly, the KEGG pathway analysis showed that the over-represented terms are enriched in many aspects of hepatic metabolic pathways (Figure 6B), which are consistent with the recent reports that focus on the relationship between HBV-related liver diseases and the derangement of hepatic metabolic pathways [31, 32]. However, these predicted functions of IncRNAs require experimental verification. Furthermore, whether the deregulated expression of IncRNAs by HBV may benefit HBV own replication will be investigated in the future in our lab.

A known lncRNA, n346077, encodes a 2609bp transcript and is located in the opposite strand of MRPL23 gene, was one of the most down-regulated lncRNA in HBV-related HCC. To test whether it contribute to the pathogenesis of HCC, we examined its effect on the

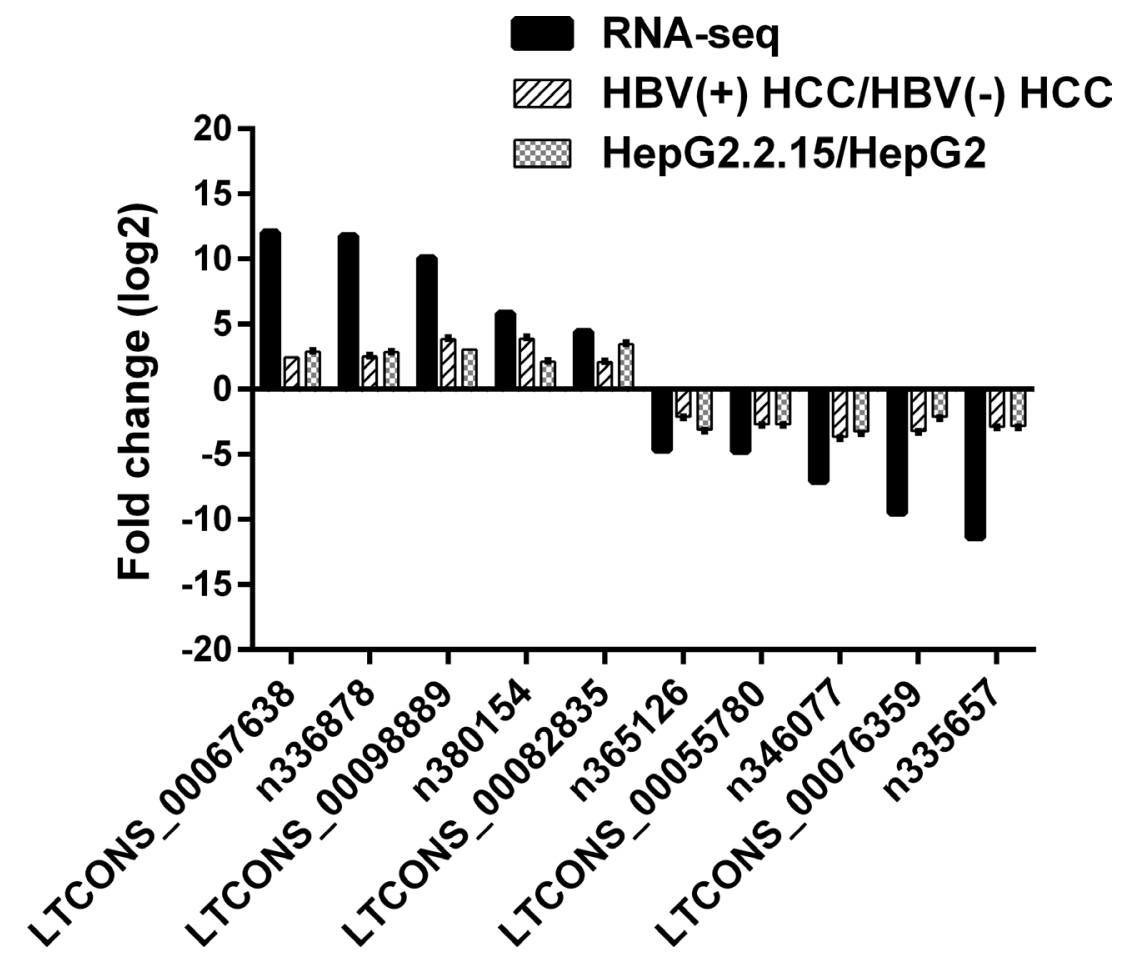

Figure 4: Validation for the expression of 10 randomly selected IncRNAs using RT-qPCR. RT-qPCR analysis of RNA extracted from $10 \mathrm{HBV}(+) \mathrm{HCC}$ patients and $3 \mathrm{HBV}(-) \mathrm{HCC}$ patients or HepG2.2.15 and HepG2 cells. $\beta$-Actin was used as an internal control. Each sample was analyzed in triplicate. The heights of column represent mean fold changes (log2 transformed) compared with control groups. 


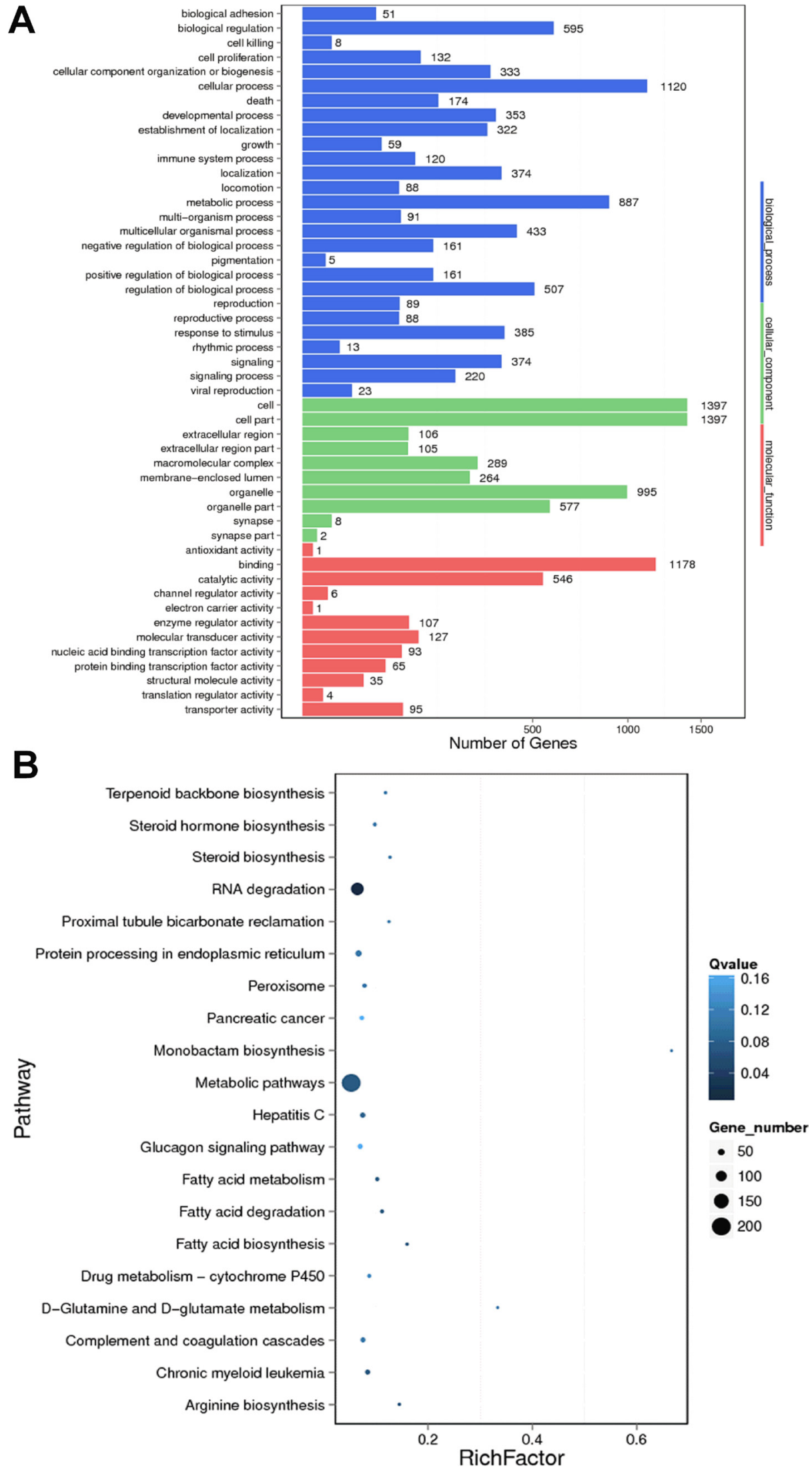

Figure 5: GO and KEGG pathway analysis of differentially expressed mRNAs between HBV (+) HCC and HBV (-) HCC tissue samples. (A) The Go terms covering domains of biological processes, cellular components and molecular functions enriched among up- and down-regulated mRNAs. (B) Top 20 enriched pathways among up- and down-regulated mRNAs. 


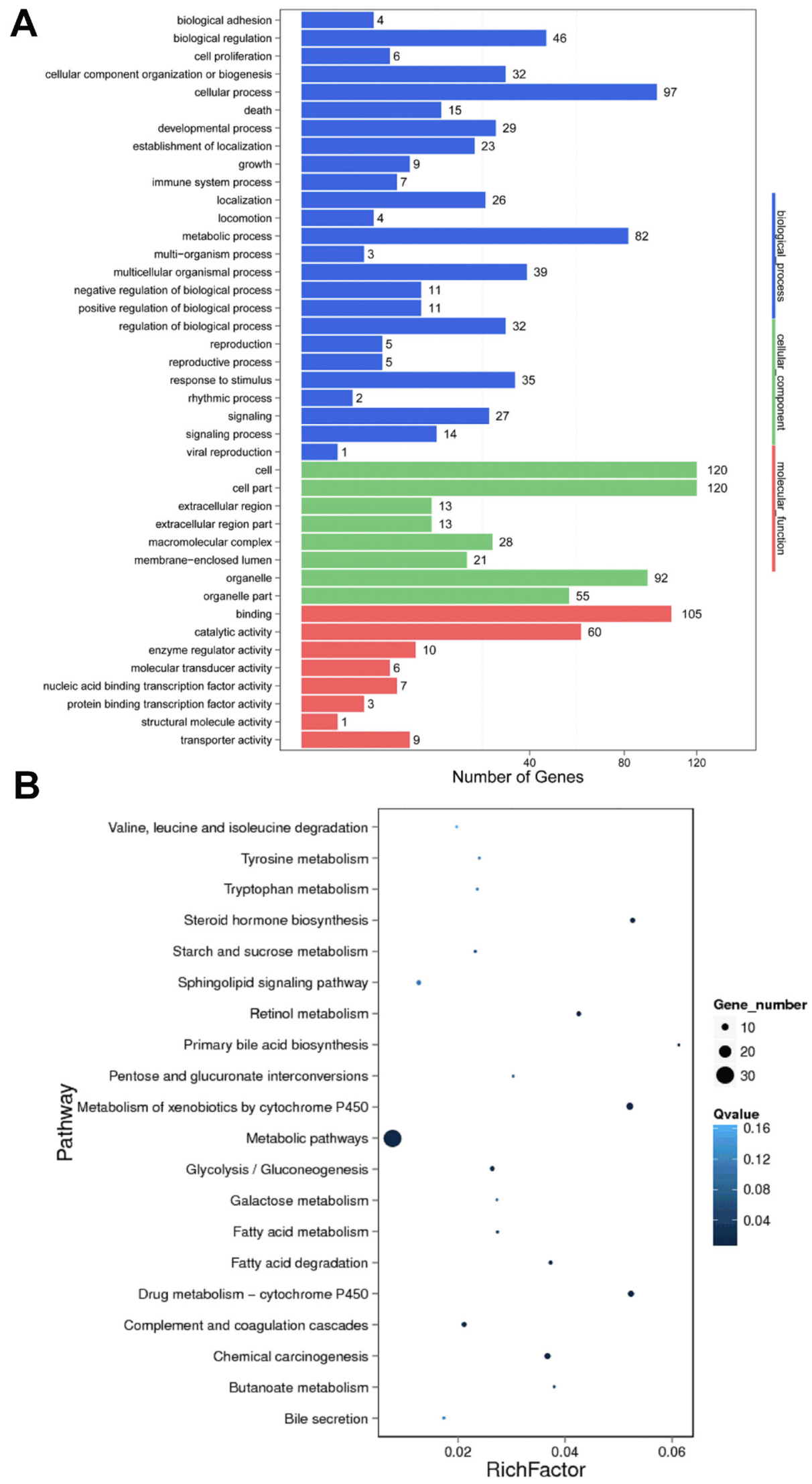

Figure 6: GO and KEGG pathway analysis of target genes of differentially expressed IncRNAs between HBV (+) HCC and HBV (-) HCC tissue samples. (A) GO analysis of predicted cis-regulated target protein-coding genes of differentially expressed IncRNAs. (B) KEGG pathway analysis of predicted cis-regulated target protein-coding genes of differentially expressed lncRNAs. 
malignant behavior of hepatomas cells. MTT and colony formation assays showed no significant differences when the expression level of n346077 was changed. Tranwell assays with or without matrigel showed that n346077 overexpression attenuated but n346077 knockdown enhanced the invasion and migration abilities of HepG2 and QGY-7703 cells, revealing that n346077 may act as a tumor suppressor gene in hepatoma cell lines, which explain its decreased expression in HBV-related HCC. It has been reported that MRPL23 can suppress the proliferation of gastric cancer cells and enhance the therapeutic efficacy of adenoviral-mediated p53 gene transfer in models of human gastric cancer by inhibiting MDM2-mediated p53 degradation [33-35]. Since n346077 is located in the opposite strand of MRPL23 gene, whether it functions through augmenting MRPL23 tumor suppressor function will be investigated in the future.

In conclusion, we first profile the lncRNAs expression in HBV-related HCC based on transcriptome RNA-seq approach and provide novel insight into the pathogenesis of HBV-related HCC. Further exploration of the lncRNAs function will be helpful in our understanding the regulatory roles of lncRNAs in HBV-related HCC development.

\section{MATERIALS AND METHODS}

\section{Patients and tissue samples}

Tissues samples from $3 \mathrm{HBV}(+) \mathrm{HCC}$ patients and $3 \mathrm{HBV}(-) \mathrm{HCC}$ patients were used for deep sequencing. Tissues samples from $10 \mathrm{HBV}(+) \mathrm{HCC}$ patients and 3 HBV (-) HCC patients were used for data validation. The liver cancer tissues were snap-freezed in liquid nitrogen immediately after removal and then stored at $-80^{\circ} \mathrm{C}$ before RNA extraction. Written informed consent was obtained from all patients and the study was approved by the Ethics Committee of Tianjin Medical University. Detailed information of the patients is listed in Table 1.
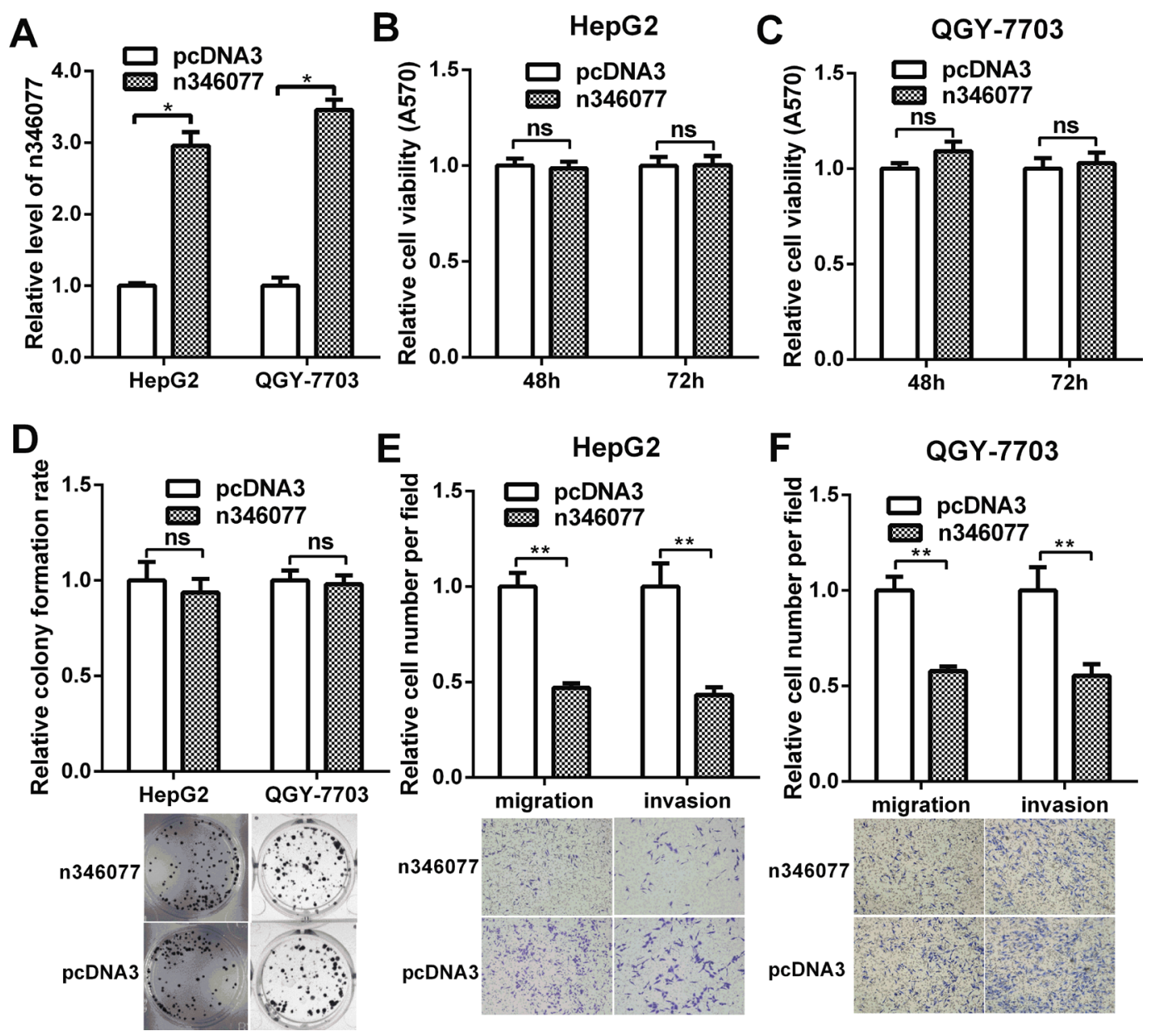

Figure 7: The effect of $\mathbf{n} 346077$ overexpression on malignant behavior of HCC cells in vitro. (A) The expression level of n346077 was analyzed by RT-qPCR in HepG2 and QGY-7703 cells after transfection with n346077 overexpression plasmid. (B) and (C) Cell viability was measured in both HepG2 and QGY-7703 cells at 48 h, $72 \mathrm{~h}$ after transfection with n346077 overexpression plasmid by MTT assay. (D) Colony formation assay was performed in both HepG2 and QGY-7703 cells after transfection with n346077. Representative images are shown. (E) and (F) The migration and invasion abilities were determined both in HepG2 and QGY-7703 cells after transfection with $\mathrm{n} 346077$ by Transwell migration and invasion assays. Cells in three random fields of view at $100 \times$ magnification were counted and expressed as the average number of cells per field. Representative images are shown. ${ }^{*} P<0.05 ; * * P<0.01$. 


\section{RNA extraction and sequencing}

Total RNA was extracted from tissue samples using TRIzol reagent (Invitrogen, MA, USA) according to the manufacturer's protocol. The preparation of whole transcriptome libraries and deep sequencing were carried out at the Beijing Genomic Institute at Shenzhen (BGIShenzhen, Shenzhen, China). RNA-Seq was performed on an Illumina Hiseq 2000 platform and 90 bp paired-end reads were generated according to Illumina's protocol.

\section{RNA-Seq data analysis}

Raw data were first processed using in-house Perl scripts. In this step, clean data were obtained by removing reads containing adapter, reads containing $\mathrm{N}$ for more than $10 \%$ of all the bases and low quality reads.

The paired-end clean reads were then aligned to human genome build 19 (hg19) with HISAT, The mapped reads of each sample was assembled using Cufflinks (v2.1.1) in a reference-based approach. For mRNA analyses, the RefSeq database (Build 38) was chosen as the annotation references. For lncRNA analyses, the NONCODE v3 database was chosen as the annotation references.

\section{Differential expression analysis}

The expression level of each transcript was calculated with RSEM and was expressed as FPKM (Fragments Per $\mathrm{Kb}$ per Million reads). Volcano Plot, scatter plot filtering and hierarchical clustering were performed to identify differentially expressed lncRNAs and mRNAs with statistical significance. The threshold is Fold Change $\geq 2.0, P$ value $\leq 0.05$.

\section{Prediction of the function of IncRNAs}

To explore the functions of IncRNAs, we predict their target genes in cis and in trans. In cis refers to lncRNAs' action on neighboring target genes. In trans refers to the influence of lncRNAs on other genes at the expression level. In present study, we defined the mRNAs as cis-
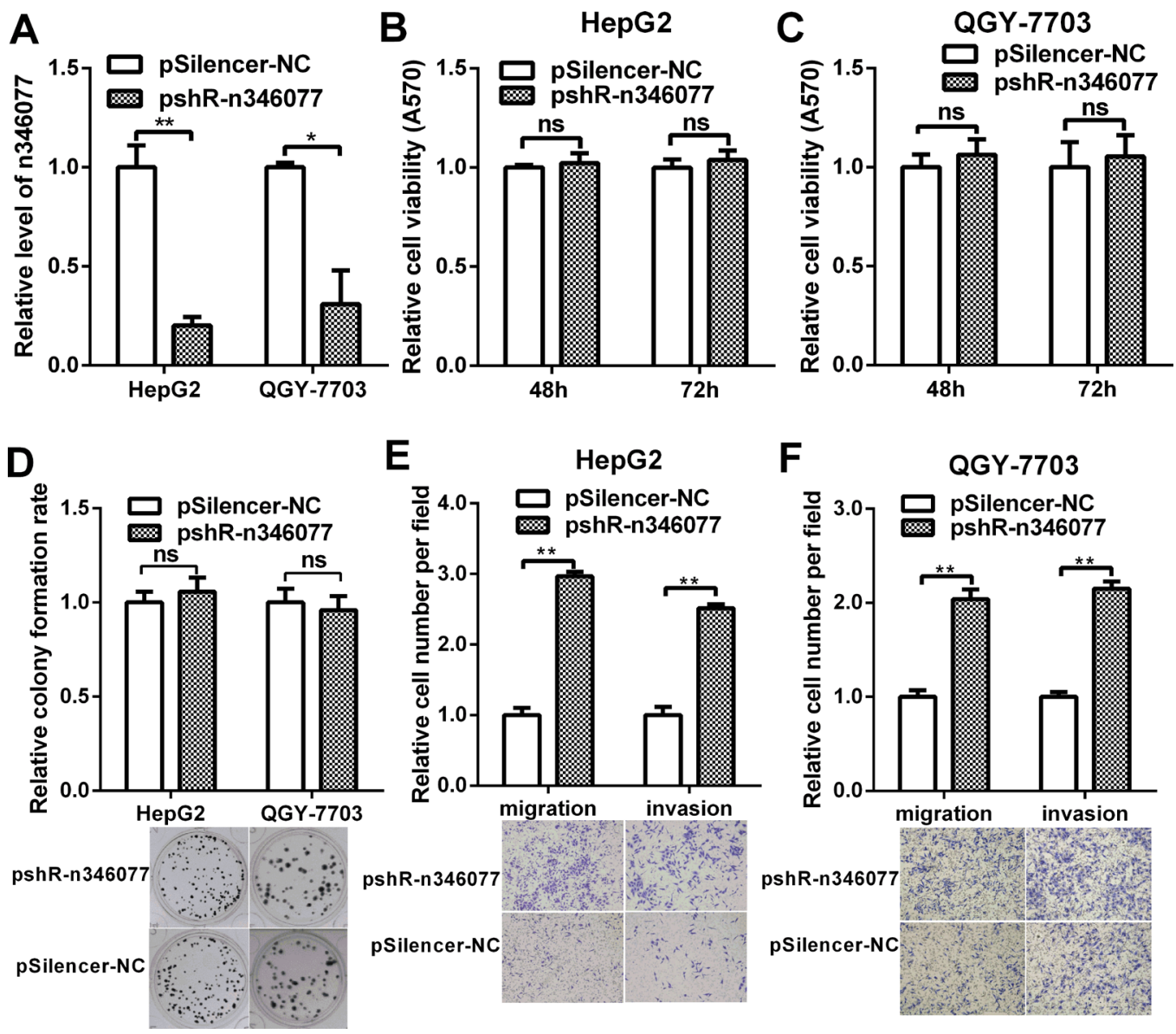

Figure 8: The effect of $\mathbf{n} 346077$ knockdown on malignant behavior of HCC cells in vitro. (A) The expression level of n346077 was analyzed by RT-qPCR in HepG2 and QGY-7703 cells after transfection with pshR-n346077. (B) and (C) MTT assay of both HepG2 and QGY-7703 cells at $48 \mathrm{~h}, 72 \mathrm{~h}$ after transfection with pshR-n346077. (D) Colony formation assay was performed in both HepG2 and QGY-7703 cells after transfection with pshR-n346077. Representative images are shown. (E) and (F) Transwell migration and invasion assays were performed in both HepG2 and QGY-7703 cells after transfection with pshR-n346077. Representative images are shown. ${ }^{*} P<0.05 ; * * P<0.01$. 
Table 1: The clinical and pathological features of the liver cancer patients

ID
\begin{tabular}{|l|c|c|c|c|}
\hline Sex & age & HBV DNA & Tumor \\
Tumors used for sequencing $(n=6)$ & & & & \\
\hline HBV $(-)$ HCC & & & - & T1N0M0 \\
\hline N1 & male & 38 & - & T1N0M0 \\
\hline N2 & male & 34 & - & T1N0M0 \\
\hline N3 & male & 34 & & \\
\hline HBV $(+)$ HCC & & & $3.56 * 10 \mathrm{E} 4$ & T1N0M0 \\
\hline P1 & male & 30 & $1.41 * 10 \mathrm{E} 4$ & T1N0M0 \\
\hline P2 & male & 46 & $1.05 * 10 \mathrm{E} 7$ & T3N0M0 \\
\hline P3 & male & 36 & & \\
\hline Tumors used for validation $(n=13)$ & & & & \\
\hline HBV $(+)$ HCC & & & $1.04 * 10 \mathrm{E} 6$ & T3N0M0 \\
\hline V1 & male & 40 & $4.41 * 10 \mathrm{E} 4$ & T1N0M0 \\
\hline V2 & male & 42 & $1.17 * 10 \mathrm{E} 6$ & T4N0M0 \\
\hline V3 & male & 55 & $6.12 * 10 \mathrm{E} 3$ & T3N0M0 \\
\hline V4 & female & 65 & $1.21 * 10 \mathrm{E} 4$ & T4N0M0 \\
\hline V5 & female & 51 & $2.86 * 10 \mathrm{E} 3$ & T2N0M0 \\
\hline V6 & male & 36 & $6.37 * 10 \mathrm{E} 5$ & T4NOMO \\
\hline V7 & male & 50 & $1.35^{*} 10 \mathrm{E} 3$ & T4NOMO \\
\hline V8 & male & 53 & $4.15 * 10 \mathrm{E} 4$ & T1N0M0 \\
\hline V9 & male & 41 & $1.63 * 10 \mathrm{E} 4$ & T1N0MO \\
\hline V10 & male & 69 & & \\
\hline HBV $(-)$ HCC $(n=3$, the tissues for & & & & \\
\hline sequencing) & & & & \\
\hline
\end{tabular}

regulated target genes when (1) it was located within $10 \mathrm{~kb}$ upstream or $100 \mathrm{~kb}$ downstream in genomic distance from the lncRNA and (2) both Spearman correlation coefficient (SCC) and Pearson correlation coefficient $(\mathrm{PCC}) \geq 0.8$. We defined the mRNAs as trans-regulated target genes when (1) it is located beyond the above distance in genomic distance from the IncRNA or in different chromosomes.

\section{GO analysis and KEGG pathway analysis}

GO enrichment analyses of differentially expressed genes or lncRNA target genes were implemented by the GO seq $\mathrm{R}$ package, in which gene length bias was corrected. KEGG pathway analyses were performed using KOBAS software.

\section{RT-qPCR}

Total RNA was isolated using Trizol (Invitrogen, MA, USA). First strand cDNA was synthesized using Moloney murine leukemia virus reverse transcriptase (Promega, Madison, WI, USA). RT-qPCR analysis was performed with the SYBR Premix Ex TaqTM kit (TaKaRa,
Shiga, Japan) according to the manufacturer's instructions. Samples were normalized using $\beta$-actin as an endogenous control. All the primers used are listed in Table 2.

\section{Cell culture and transfection}

The human hepatoma cell line HepG2 cells and QGY-7703 cells were cultured in Dulbecco's modified Eagle's medium (DMEM) supplemented with 10\% fetal bovine serum at $37^{\circ} \mathrm{C}$ in a humidified atmosphere with $5 \% \mathrm{CO}_{2}$. Cells were transfected with plasmids using Lipofectamine $^{\mathrm{TM}} 2000$ (Invitrogen, MA, USA) following the manufacturer's protocol.

\section{Plasmids construction}

The pcDNA3.0 was used to generate n346077 overexpressing plasmid with method as described previously [36]. Briefly, the cDNA of n346077 was amplified from the cDNA of HepG2 cells and then cloned into the KpnI and EcoRI restriction sites of pcDNA3.0 (named n346077). The shRNA oligonucleotides of n346077 was annealed and cloned into pSilencer 2.1-U6 
Table 2: Primers used in this work

\begin{tabular}{|c|c|}
\hline primers & sequences $\left(5^{\prime}\right.$ to $\left.3^{\prime}\right)$ \\
\hline LTCONS_00067638 forward & GAGCCTGTTGTCCTTTGATTGA \\
\hline LTCONS_00067638 reverse & TTCTGGGAAATAGTGCTTGATA \\
\hline LTCONS_00098889 forward & TCACCTGTCAAATCTTCCTCAA \\
\hline LTCONS_00098889 reverse & TGTCTGCCACATCATTCCTTAT \\
\hline LTCONS_00082835 forward & TCTCCGTAGGTTCTCCAATG \\
\hline LTCONS_00082835 reverse & TGGAATGGGATGTGCTGAAT \\
\hline LTCONS_00055780 forward & ATACATAAAGAACAATGAGGGAGC \\
\hline LTCONS_00055780 reverse & TTCACGACAAACAGACGAACAC \\
\hline LTCONS_00076359 forward & TCCACTGGCATTCTATCACCTA \\
\hline LTCONS_00076359 reverse & GAAGGGATAAGCCTCTGGTCTC \\
\hline n336878 forward & CTCCATGTAGACTGTGCTCG \\
\hline n336878 reverse & AGGGCTTGAGTGGATGGGAATA \\
\hline n380154 forward & GAGAAATGAAAAGGGAGGTC \\
\hline n380154 reverse & TTGTATCGGGCAAAGGTG \\
\hline n365126 forward & GGTACTATCAATGGAGGTGGA \\
\hline n365126 reverse & GATTTCGTGCTTAGTTGCTTTT \\
\hline n346077 forward & CCCACACACTAGCCCACTGTTC \\
\hline n346077 reverse & CATGTCCGTGCCAATTCCTCAA \\
\hline n335657 forward & CGACATGACCACCTTCAGCAAG \\
\hline n335657 reverse & GTAAGCGTAGCGTTCACCAGAT \\
\hline n346077-sense & CGCGGTACCGCCCTCACTCCCCAGGCCCA \\
\hline n346077-antisense & CCGGAATTCCCCCACCAGGGGCTGTGCA \\
\hline n346077-shR-top & $\begin{array}{l}\text { GATCCGACCCTGTGTCTGGAATGTGGCTCG } \\
\text { AGCCACATTCCAGACACAGGGTCTTTTTGA }\end{array}$ \\
\hline n346077-shR-bot & $\begin{array}{l}\text { AGCTTCAAAAAGACCCTGTGTCTGGAATGTGG } \\
\text { CTCGAGCCACATTCCAGACACAGGGTCG }\end{array}$ \\
\hline
\end{tabular}

neo vector at BamHI and HindIII sites to construct the knockdown plasmids pshR-n346077. The insertions were confirmed by DNA sequencing. The primers and oligonucleotides used are listed in Table 2.

\section{Cell viability and colony formation assay}

For cell viability assay, HepG2 cells or QGY-7703 cells transfected with n346077 or pshR-n346077 were seeded in 96-well plates and tested using the MTT assay at different time points. For colony formation assay, the transfected HepG2 cells or QGY-7703 cells were seeded in 12-well plates and they were stained with crystal violet and counted when most of the colonies contained at least 50 cells.

\section{Cell migration and invasion assays}

The 24-well Transwell chamber inserts with 8- $\mu \mathrm{m}$ pore size polycarbonate membrane (Corning, Cambridge, MA) without or with Matrigel (for invasion) was used to analyze the migration and invasion of tumor cells. The assays were performed as described previously [36].

\section{Abbreviations}

DMEM, Dulbecco's modified Eagle's medium; DREH, down-regulated expression by HBx; GO, gene ontology; HBx-LINE1, HBx-interspersed nuclear element 1; HCC, Hepatocellular carcinoma; HEIH, high expression in HCC; HULC, highly up-regulated in liver cancer; KEGG, Kyoto Encyclopedia of Genes and Genomes; lncRNAs, long non-coding RNAs; MRPL23, mitochondrial ribosomal protein L23; MVIH, microvascular invasion in HCC; RNA-Seq, RNA sequencing; RT-qPCR, quantitative real-time PCR.

\section{Authors' contributions}

Conception and design the experiments: H Tang. Data collection, manuscript writing: HX Fan, H Tang. 
Performed the experiments: HX Fan, QG Zhang, XP Zhao, $\mathrm{P}$ Lv, Analysis and/or interpretation of data: HX Fan, M Liu, H Tang.

\section{CONFLICTS OF INTEREST}

The authors declare no conflicts of interest.

\section{FUNDING}

This study was supported by the National Natural Science Foundation of China $(81572790 ; 81601763$; 91629302) and the Natural Science Foundation of Tianjin (16JCQNJC10500; 12JCZDJC25100).

\section{REFERENCES}

1. Torre LA, Bray F, Siegel RL, Ferlay J, Lortet-Tieulent J, Jemal A. Global cancer statistics, 2012. CA Cancer J Clin. 2015; 65:87-108.

2. Nguyen VT, Law MG, Dore GJ. Hepatitis B-related hepatocellular carcinoma: epidemiological characteristics and disease burden. J Viral Hepat. 2009; 16:453-463.

3. de Oliveria Andrade LJ, D’Oliveira A, Melo RC, De Souza EC, Costa Silva CA, Paraná R. Association between hepatitis $\mathrm{C}$ and hepatocellular carcinoma. J Glob Infect Dis. 2009; 1:33-37.

4. Sidharthan S, Kottilil S. Mechanisms of alcohol-induced hepatocellular carcinoma. Hepatol Int. 2014; 8:452-457.

5. Rieswijk L, Claessen SM, Bekers O, van Herwijnen M, Theunissen DH, Jennen DG, de Kok TM, Kleinjans JC, van Breda SG. Aflatoxin B1 induces persistent epigenomic effects in primary human hepatocytes associated with hepatocellular carcinoma. Toxicology. 2016; 350-352:31-39.

6. Gozuacik D, Murakami Y, Saigo K, Chami M, Mugnier C, Lagorce D, Okanoue T, Urashima T, Bréchot C, PaterliniBréchot P. Identification of human cancer-related genes by naturally occurring hepatitis B virus DNA tagging. Oncogene. 2001; 20:6233-6240.

7. Levrero M, Zucman-Rossi J. Mechanisms of HBV-induced hepatocellular carcinoma. J Hepatol. 2016; 64:S84-101.

8. Derrien T, Johnson R, Bussotti G, Tanzer A, Djebali S, Tilgner H, Guernec G, Martin D, Merkel A, Knowles DG, Lagarde J, Veeravalli L, Ruan X, et al. The GENCODE v7 catalog of human long noncoding RNAs: analysis of their gene structure, evolution, and expression. Genome Res. 2012; 22:1775-1789.

9. St Laurent G, Wahlestedt C, Kapranov P. The Landscape of long noncoding RNA classification. Trends Genet. 2015; 31:239-251.

10. Quinn JJ, Chang HY. Unique features of long non-coding RNA biogenesis and function. Nat Rev Genet. 2016; 17:47-62.

11. Huarte M. The emerging role of lncRNAs in cancer. Nat Med. 2015; 21:1253-1261.
12. Li C, Chen J, Zhang K, Feng B, Wang R, Chen L. Progress and Prospects of Long Noncoding RNAs (lncRNAs) in Hepatocellular Carcinoma. Cell Physiol Biochem. 2015; 36:423-434.

13. Moyo B, Nicholson SA, Arbuthnot PB. The role of long non-coding RNAs in hepatitis B virus-related hepatocellular carcinoma. Virus Res. 2016; 212:103-113.

14. Du Y, Kong G, You X, Zhang S, Zhang T, Gao Y, Ye L, Zhang X. Elevation of highly up-regulated in liver cancer (HULC) by hepatitis B virus X protein promotes hepatoma cell proliferation via down-regulating p18. J Biol Chem. 2012; 287:26302-26311.

15. Yang F, Zhang L, Huo XS, Yuan JH, Xu D, Yuan SX, Zhu N, Zhou WP, Yang GS, Wang YZ, Shang JL, Gao CF, Zhang FR, et al. Long noncoding RNA high expression in hepatocellular carcinoma facilitates tumor growth through enhancer of zeste homolog 2 in humans. Hepatology. 2011; 54:1679-1689.

16. Lau CC, Sun T, Ching AK, He M, Li JW, Wong AM, Co NN, Chan AW, Li PS, Lung RW, Tong JH, Lai PB, Chan HL, et al. Viral-human chimeric transcript predisposes risk to liver cancer development and progression. Cancer Cell. 2014; 25:335-349.

17. Iizuka N, Oka M, Yamada-Okabe H, Mori N, Tamesa T, Okada T, Takemoto N, Tangoku A, Hamada K, Nakayama H, Miyamoto T, Uchimura S, Hamamoto Y. Comparison of gene expression profiles between hepatitis B virus- and hepatitis $\mathrm{C}$ virus-infected hepatocellular carcinoma by oligonucleotide microarray data on the basis of a supervised learning method. Cancer Res. 2002; 62:3939-3944.

18. Zhang H, Diab A, Fan H, Mani SK, Hullinger R, Merle P, Andrisani O. PLK1 and HOTAIR accelerate proteasomal degradation of SUZ12 and ZNF198 during hepatitis B virus-induced liver carcinogenesis. Cancer Res. 2015; 75:2363-2374.

19. Lv J, Fan HX, Zhao XP, Lv P, Fan JY, Zhang Y, Liu M, Tang H. Long non-coding RNA Unigene56159 promotes epithelial-mesenchymal transition by acting as a ceRNA of miR-140-5p in hepatocellular carcinoma cells. Cancer Lett. 2016; 382:166-175.

20. Huang JF, Guo YJ, Zhao CX, Yuan SX, Wang Y, Tang GN, Zhou WP, Sun SH. Hepatitis B virus X protein $(\mathrm{HBx})-$ related long noncoding RNA (lncRNA) down-regulated expression by HBx (Dreh) inhibits hepatocellular carcinoma metastasis by targeting the intermediate filament protein vimentin. Hepatology. 2013; 57:1882-1892.

21. Yuan SX, Yang F, Yang Y, Tao QF, Zhang J, Huang G, Yang Y, Wang RY, Yang S, Huo XS, Zhang L, Wang F, Sun $\mathrm{SH}$, et al. Long noncoding RNA associated with microvascular invasion in hepatocellular carcinoma promotes angiogenesis and serves as a predictor for hepatocellular carcinoma patients' poor recurrence-free survival after hepatectomy. Hepatology. 2012; 56:2231-2241.

22. Pan YF, Qin T, Feng L, Yu ZJ. Expression profile of altered long non-coding RNAs in patients with HBV-associated 
hepatocellular carcinoma. J Huazhong Univ Sci Technolog Med Sci. 2013; 33:96-101.

23. Yu TT, Xu XM, Hu Y, Deng JJ, Ge W, Han NN, Zhang MX. Long noncoding RNAs in hepatitis B virus-related hepatocellular carcinoma. World J Gastroenterol. 2015; 21:7208-7217.

24. Gong X, Wei W, Chen L, Xia Z, Yu C. Comprehensive analysis of long non-coding RNA expression profiles in hepatitis B virus-related hepatocellular carcinoma. Oncotarget. 2016; 7:42422-42430. doi: 10.18632/ oncotarget.9880.

25. Niu J, Lin Y, Liu P, Yu Y, Su C, Wang X. Microarray analysis on the lncRNA expression profile in male hepatocelluar carcinoma patients with chronic hepatitis B virus infection. Oncotarget. 2016; 7:76169-76180. doi: 10.18632/oncotarget.12732.

26. Hu B, Huo Y, Chen G, Yang L, Wu D, Zhou J. Functional prediction of differentially expressed lncRNAs in HSV-1 infected human foreskin fibroblasts. Virol J. 2016; 13:137.

27. Esclatine A, Taddeo B, Roizman B. The UL41 protein of herpes simplex virus mediates selective stabilization or degradation of cellular mRNAs. Proc Natl Acad Sci USA. 2004; 101:18165-18170.

28. Taddeo B, Zhang W, Roizman B. The UL41 protein of herpes simplex virus 1 degrades RNA by endonucleolytic cleavage in absence of other cellular or viral proteins. Proc Natl Acad Sci USA. 2006; 103:2827-2832.

29. Geng M, Xin X, Bi LQ, Zhou LT, Liu XH. Molecular mechanism of hepatitis $\mathrm{B}$ virus $\mathrm{X}$ protein function in hepatocarcinogenesis. World J Gastroenterol. 2015; 21:10732-10738.
30. Yang X, Wu L, Lin J, Wang A, Wan X, Wu Y, Robson SC, Sang X, Zhao H. Distinct hepatitis B virus integration patterns in hepatocellular carcinoma and adjacent normal liver tissue. Int J Cancer. 2017; 140:1324-1330.

31. Shi YX, Huang CJ, Yang ZG. Impact of hepatitis B virus infection on hepatic metabolic signaling pathway. World J Gastroenterol. 2016; 22:8161-8167.

32. Hou Q, Duan ZJ. Metabonomic window into hepatitis B virus-related hepatic diseases. World J Hepatol. 2016; 8:1-8.

33. Jin A, Itahana K, O'Keefe K, Zhang Y. Inhibition of HDM2 and activation of p53 by ribosomal protein L23. Mol Cell Biol. 2004; 24:7669-7680.

34. Zhang Y, Shi Y, Li X, Du W, Luo G, Gou Y, Wang X, Guo X, Liu J, Ding J, Wu K, Fan D. Inhibition of the p53MDM2 interaction by adenovirus delivery of ribosomal protein L23 stabilizes p53 and induces cell cycle arrest and apoptosis in gastric cancer. J Gene Med. 2010; 12:147-156.

35. Zhang YF, Zhang BC, Zhang AR, Wu TT, Liu J, Yu LF, Wang WX, Gao JF, Fang DC, Rao ZG. Co-transduction of ribosomal protein L23 enhances the therapeutic efficacy of adenoviral-mediated p53 gene transfer in human gastric cancer. Oncol Rep. 2013; 30:1989-1995.

36. Liu Q, Guo X, Que S, Yang X, Fan H, Liu M, Li X, Tang H. LncRNA RSU1P2 contributes to tumorigenesis by acting as a ceRNA against let-7a in cervical cancer cells. Oncotarget. 2016; 8:43768-43781. doi: 10.18632/oncotarget.10844. 